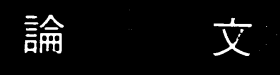

\title{
Auto-Controlled Mechanism for Rheological Properties of Native Ovalbumin Aqueous Colloid
}

\author{
Takayoshi Matsumoto* and Jiro Chiba \\ Department of Polymer Chemistry, Kyoto University, \\ Kyoto 606, Japan
}

（原稿受理：1991年 4 月 1 日）

\begin{abstract}
Native ovalbumin (OA) molecules in aqueous colloids are more stable in an ordered arrangement state than in a random dispersion state. Rate of the stabilization from the random dispersion state to the ordered arrangement state is extremely accelerated by shear flow. Isotropic colloids of native OA molecules in buffer solutions show a remarkable yield stress $\sigma_{\mathrm{y}}$ and a rigidity $G$ even in very dilute systems such as 0.027 wt $\%$. The values of $\sigma_{\mathrm{y}}$ and $G$ remain almost constant over a wide concentration range from 0.1 to $17 \%$. This suggests that the systems are spontaneously controlled (autocontrolled) in order to suppress the increase in $\sigma_{\mathrm{y}}$ and $\mathrm{G}$ with increasing concentration of OA. This auto-controlled mechanism disappears completely with gelation by heat denaturation.
\end{abstract}

Key words: Protein Colloid / Ovalbumin / Rigidity of Colloid / Yield Stress / DLVO Theory

\section{INTRODUGTION}

Ovalbumin $(\mathrm{OA})$ is a globular protein which is composed of a single polypeptide chain with 385 amino residues. The complete sequence of the amino acids in the OA molecule was determined by McReynolds et al. and Nisbet et $\mathrm{al}^{13,2)}$. On the other hand, relatively little is known of the secondary and tertiary structure. Helical content of the OA which is measured with optical rotatory dispersion depends on the calculated methods and scatters in a range from $17 \%$ to $34 \%^{3) \sim 6)}$.

It has been considered that the native OA molecule disperses in a stable globular form in aqueous systems. In the previous study ${ }^{7}$, we investigated on the shape and the dispersed state of the OA molecule in phosphate buffer solution, through small angle X-ray scattering (SAXS) measurement and obtained the following results. The OA molecule is almost spherical particle with a radius of ca. $25.2 \AA$, and disperses in the monomeric state at a concentration range of less than $0.1 \%$ and in the dimeric state at a range of $0.5 \sim 1 \%$. The native OA molecules disperse having a certain solid-like structure with ordered arrangement in sodium phosphate buffer solution $(\mathrm{pH} 7.0)$ over a wide concentration range of $0.03 \mathrm{wt} \sim 17 \mathrm{wt} \%^{7)}$.

In the present study we intend to discuss the

\footnotetext{
* Takayoshi Matsumoto: Department of Polymer Chemistry Kyoto University, Kyoto 606 Japan.
}

dispersed state of the OA molecules at relatively high concentration region of $5 \sim 17 \mathrm{wt} \%$, which was not described explicitly in the previous paper, and discuss the concentration dependence of rheological properties of the aqueous colloids of the native $\mathrm{OA}$ molecule.

\section{EXPERIMENTAL}

\subsection{Material}

Crystallized, lyophilized ovalbumin (Sigma, grade $V$, Lot No. 19F-8105), which was electrophoretically pure, was used without further purification. The molecular weight of the OA is ca. 44000 by the SDS-polyacrylamide gel electrophoresis and the weight average molecular weight by the light scattering is $43700^{7)}$.

The isoelectric point can be determined to $\mathrm{pH}$ 4.4 at which the $\zeta$-potential is equal to zero ${ }^{8}$. This isoelectric point is almost coincident with the value reported $^{5}$.

Sample colloids were prepared in cooling condition under an ice-water bath.

\subsection{Measurements}

The light scattering was measured using a low angle laser light scattering photometer $(\lambda=6328 \AA$, Chromatix, KMX6) at scattering angle ca. $5^{\circ}$.

SAXS was measured using a $6 \mathrm{~m}$ point-focusing SAXS camera at the High Intensity X-ray Laboratory, Kyoto University. The wavelength of the X-ray is $1.542 \AA$.

Rheological measurements were carried out by 
means of a cone-plate type Weissenberg rheogoniometer (Sangamo Controls, R18) which was devised to prevent vaporization of water. The diameter and the angle of the cone used are $7.0 \mathrm{~cm}$ and $1.77^{\circ}$.

\section{RESULTS AND DISGUSSION}

\subsection{Auto-controlled property}

Figure 1 shows the weight average molecular weight $M_{\mathrm{w}}$ plotted against denaturation time at various temperatures. The molecular weight increases with association which is due to the heat denaturation of the OA molecules ${ }^{9)}$. However, the molecular weight remains almost a constant value or increases only very slightly in the early stage of the denaturation at $20^{\circ} \mathrm{C}$. Consequently, the various experiments carried out at $20^{\circ} \mathrm{C}$.

Figure 2 shows the X-ray scattering intensity $\bar{I}$ $\left(=I-I_{\mathrm{S}}\right)$ plotted against wave vector $q[=(4 \pi / \lambda)$ $\sin (\theta / 2)]$, where $I$ and $I_{\mathrm{S}}$ are the scattering intensity of the colloid system and the medium, $\theta$ : scattering angle, $\lambda$ : X-ray wavelength. The scattering curve manifests a relatively broad peak and this shows that there is no well developed crystalline structure in these systems from the X-ray scattering viewpoint. However, from the rheological viewpoint, these systems show apparent yield stress and rigidity as shown in the previous paper and also in the present paper. This means that a certain solid-like structure may exist in these systems and that the rheological properties are more sensitive than the X-ray scattering to detect the solid-like structure. According to the above consideration, it is reasonable to obtain certain information on the separation distance $d$ between the the OA molecules in the ordered arrangement from the peak position $q_{\mathrm{m}}$ (designated by an arrow) using the Bragg equation. Table I shows these quantities and the association number (degree of association)

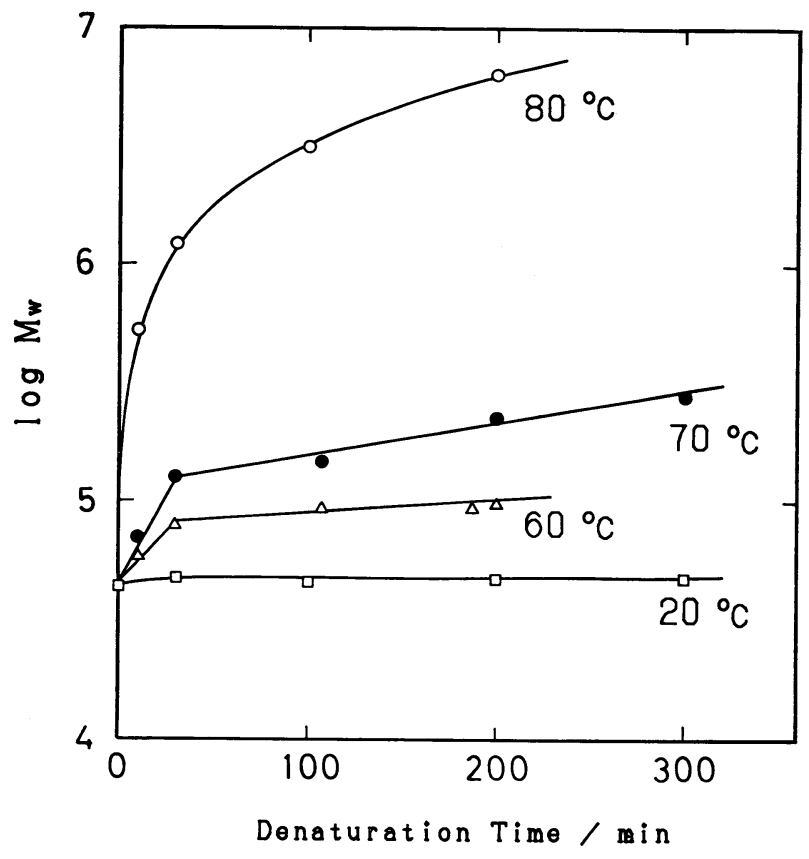

Fig. 1 Weight average molecular weight $M_{\mathrm{w}}$ plotted against denaturation time at various temperatures.

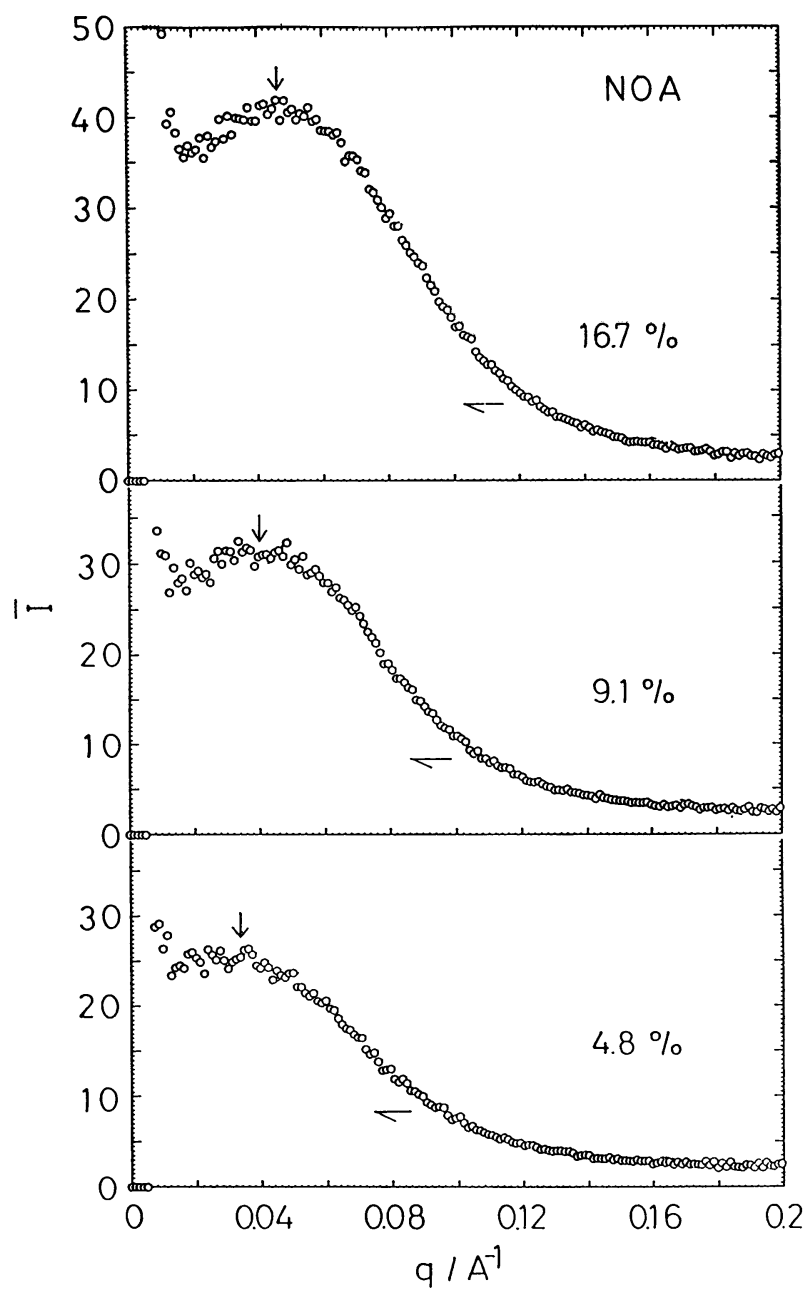

Fig. 2 Scattering intensity $\bar{I}$ in SAXS plotted against $q$ for the native $\mathrm{OA}$ aqueous colloids at concentrations of $4.8,9.1$ and $16.7 \%$

Table I. Separation distance and association number calculated from X-ray scattering.

\begin{tabular}{cccc}
\hline$c / \mathrm{wt} \%$ & $q_{\mathrm{m}} / A^{-1}$ & $d / A$ & $N_{\mathrm{as}}$ \\
\hline 4.8 & 0.0334 & 188 & 3.09 \\
9.1 & 0.0399 & 157 & 3.41 \\
16.7 & 0.0464 & 135 & 3.98 \\
\hline
\end{tabular}

Table II. Dispersing states of native ovalbumin in phosphate buffer solution

\begin{tabular}{|c|c|c|c|}
\hline$c / \mathrm{wt} \%$ & $c<0.1$ & $0.5-1$ & $4.8-16.7$ \\
\hline $\begin{array}{l}\text { dispersing } \\
\text { states }\end{array}$ & monomeric & dimeric & $\begin{array}{l}\text { tri- or } \\
\text { tetrameric }\end{array}$ \\
\hline $\begin{array}{l}\text { measuring } \\
\text { methods }\end{array}$ & $\begin{array}{l}\text { molecular } \\
\text { wt. by LS }\end{array}$ & $\begin{array}{l}\text { Guinier } \\
\text { plot in } \\
\text { SAXS }\end{array}$ & $\begin{array}{l}\text { scattering } \\
\text { peak in } \\
\text { SAXS }\end{array}$ \\
\hline
\end{tabular}

$N_{\text {as }}$ which is calculated from the separation distance $\mathrm{d}$ assuming the face centered cubic arrangement. The association numbers are about 3 to 4 at the concentration range of $5 \sim 17 \%$.

Table II shows the dispersed state of the native OA molecules in sodium phosphate buffer solution ( $\mathrm{pH} 7.0)$ with the measuring methods ${ }^{7}$. 


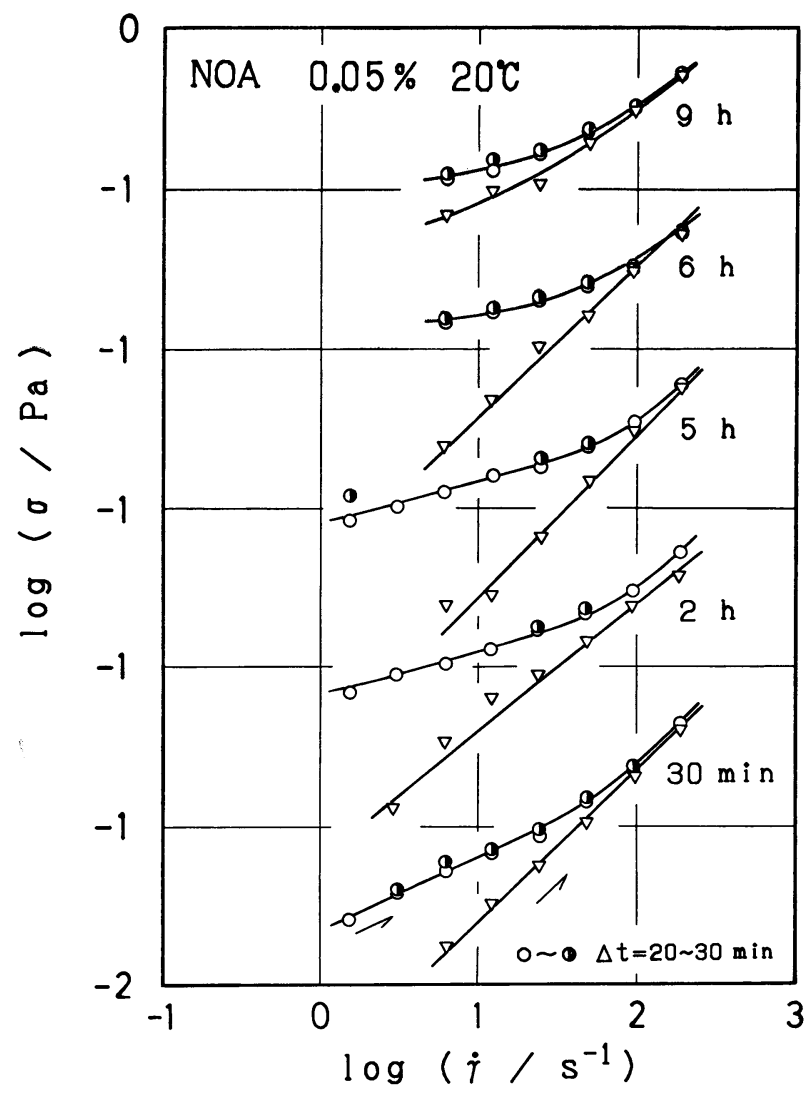

Fig. 3 Flow curves for the OA colloid of $0.05 \%$ at various times from 0.5 to 9 hours after the colloid preparation. Absolute value at each flow curve bases on the position of log $\left(\sigma / P_{\mathrm{a}}\right)=-1$ on the ordinate.

Figure 3 shows the logarithmic plots of shear stress $\sigma$ vs shear rate $\dot{\gamma}$ for the OA colloid of $0.05 \%$ at various times from 0.5 to 9 hours after the colloid preparation. The flow curve $(\sigma$ vs $\dot{\gamma})$ of the OA colloid has a shear hysteresis. The colloid prepared under about $0^{\circ} \mathrm{C}$ and $30 \mathrm{~min}$ later the first measurement (open triangle) was carried out from low to high shear rate as designated by an arrow. The system shows Newtonian flow. Immediately after cessation of the first measurement at the shear rate about $200 \mathrm{~s}^{-1}$, the second run (open circle) was started from low to high shear rate. The second run shows a typical non-Newtonian flow curve which does not depend on time, i.e. the third run (half black circle) shows substantially the same flow curve with the second run. Similar behavior can be observed for the cases in which the systems are allowed to stand at about $0^{\circ} \mathrm{C}$ for less than 6 hours after the sample preparation. However Newtonian flow cannot be detected for the first run after 9 hours standing. It should be noted that the absolute value of the shear stress, which bases on the line of $\log \sigma=-1$ on the ordinate for each measurement, is almost independent on the standing time. It is confirmed that the molecular weight of OA remains constantly during these measurements. These results mean that the transition from Newtonian to non-Newtonian flow is due to a certain ordered arrangement of OA mol-

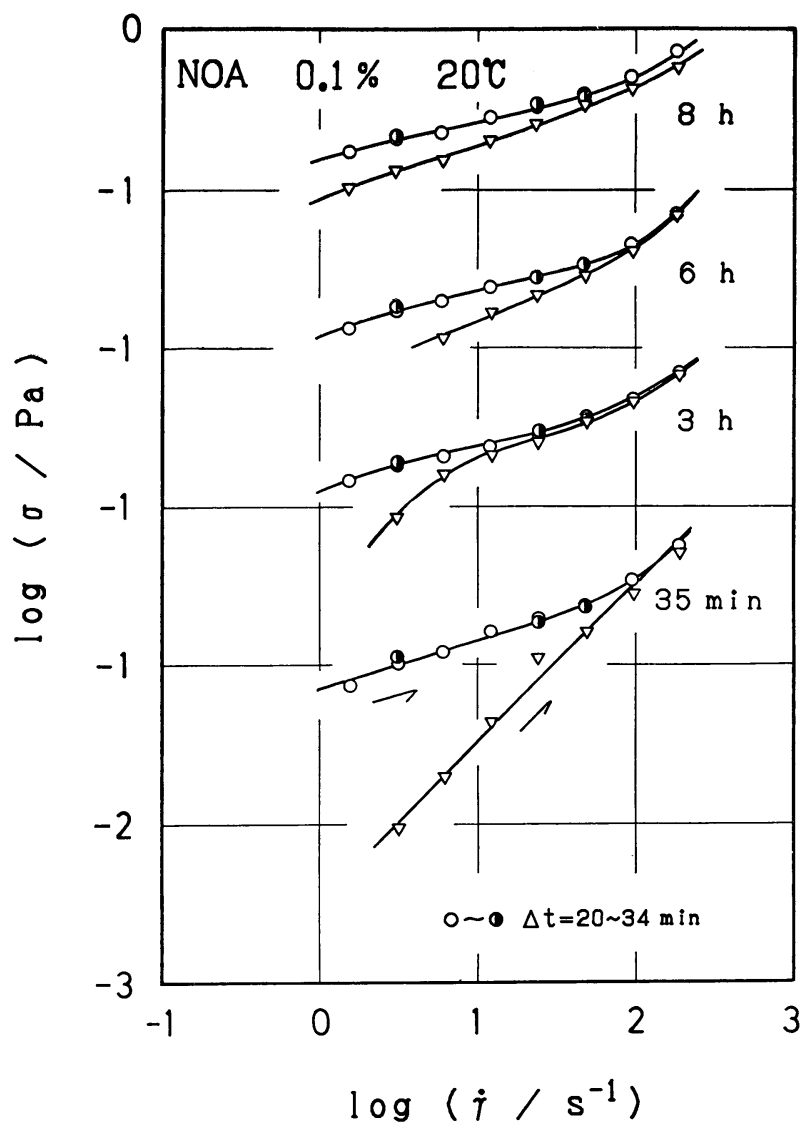

Fig. 4 Flow curves for the OA colloids of $0.1 \%$ at various times after the colloid preparation.

ecules and that the standing for about 9 hours is necessary to arrange the OA molecules, but the arrangement is remarkably accelerated by shearing. That is, the ordered arrangement is more stable state for the OA molecules in the aqueous colloid. Figure 4 shows a similar data for $0.1 \%$ colloid. The standing time needed for the ordered arrangement is only about 3 hours, i.e. the time shortens with increasing the OA concentration. Newtonian flow is no longer observed at concentrations higher than $1 \%$. According to the above consideration, it is reasonable that we use the flow curve in the stable state of the OA colloid.

Figure 5 shows the flow curves for the native OA colloids in the phosphate buffer solution ( $20 \mathrm{mM}$, $\mathrm{pH} \mathrm{7.0)}$ over a wide concentration range from 0 to $16.7 \%$. The buffer solution and the colloid of 0.017 $\%$ show Newtonian flow and the systems of higher concentrations show an apparent plateau region corresponding to yield stress. Considering that the system shows the apparent yield stress, in spite of the fact that the OA molecules disperse in the monomeric state at a concentration range of less than $0.1 \%$ as shown in Table II, the yield stress is originated from the ordered arrangement of the OA molecules. The yield stress is a certain measure of the strength of the solid structure and strongly correlates to the rigidity of the system ${ }^{10), 11)}$. There are two notable points in these flow curves. One is that the system shows relatively high yield stress even at very low concentra- 


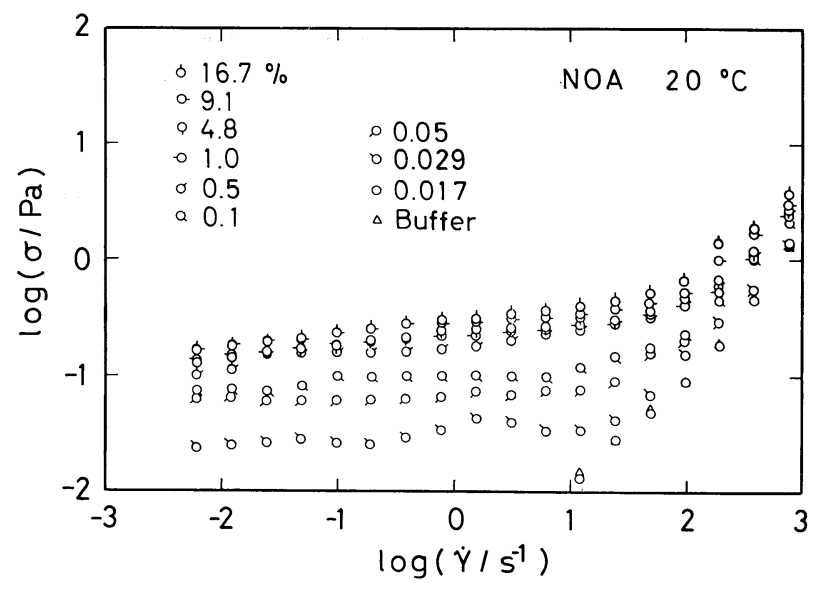

Fig. 5 Flow curves of the OA colloids in the phosphate buffer $(20 \mathrm{nM}, \mathrm{pH} 7.0)$ over a wide concentration range from 0 to $16.7 \%$.

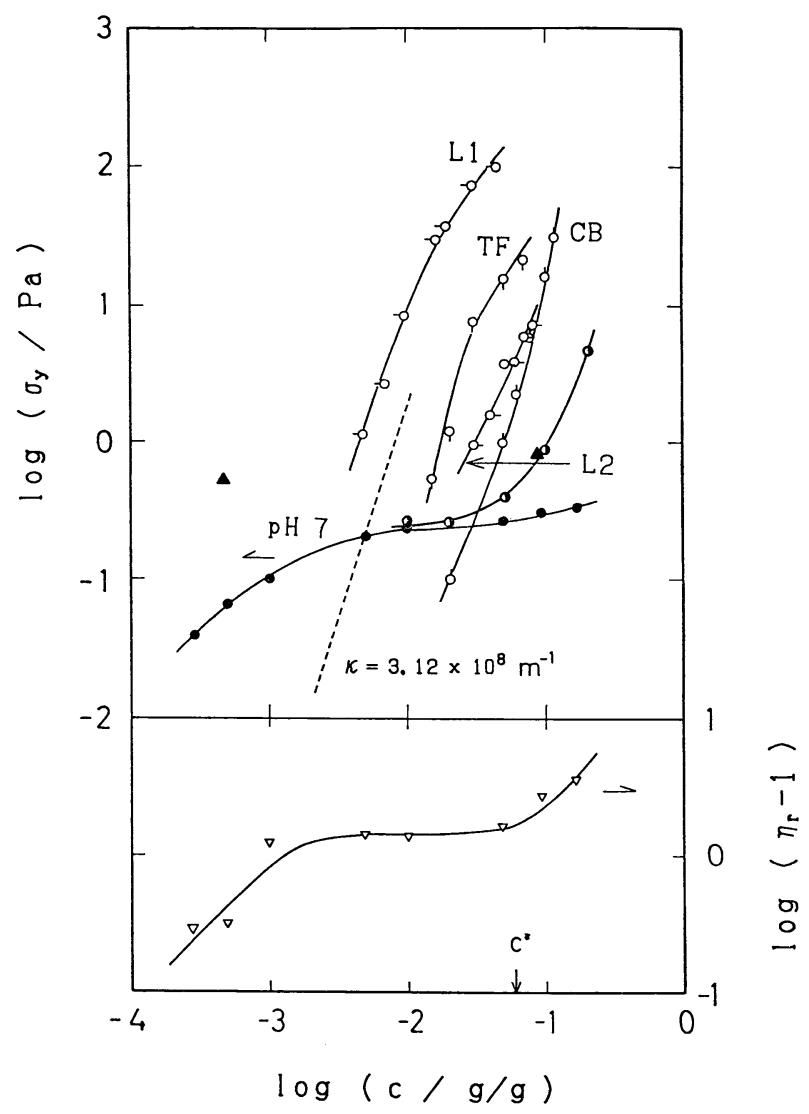

Fig. 6 Shear stress $\sigma_{\mathrm{y}}$ at $\dot{\gamma}=3 \mathrm{~s}^{-1}$ in the plateau region of the flow curve is plotted against the OA concentration $c$. Closed circle: data at $\mathrm{pH} 7.0$, closed triangle: data at $\mathrm{pH} 4,5$. half black circle: data for heat denatured system. The yield stress or the rigidity are also plotted for various colloids of polystyrene beads (L1, L2), carbon black (CB) and titanate fiber (TF).

The dashed line is the rigidity calculated by Eq. (5) using the DLVO theory for $\kappa=3.12$ $\times 10^{-8} \mathrm{~m}^{-1}$.

In the lower figure, Newtonian viscosity at high shear rate is plotted against the OA concentration. tions such as $0.03 \%$, and second is that the value of yield stress remains almost constant at high concentrations.

Figure 6 shows the shear stress (closed circle) at $\dot{\gamma}=3 \mathrm{~s}^{-1}$ in the plateau region, which is designated by $\sigma_{\mathrm{y}}$, plotted against the OA concentration c. In this figure, the yield stress and the rigidity for various colloids in the literature (open circles with pip) are also plotted against the particle concentration. For the OA colloid, $\sigma_{\mathbf{y}}$ increases in proportion to $c^{1}$ at very low concentrations where the OA molecules disperse in the monomeric state and remains almost constant (or very slightly increases) at intermediate concentration, dispersing in the dimeric state. After that, $\sigma_{\mathrm{y}}$ increases slightly in the concentration range of $5 \sim 17 \%$ where the OA molecules disperse in the trimeric to tetrameric state. As shown in the lower figure, similar behavior is observed for the Newtonian viscosity at high shear rate. Here $\eta_{\mathrm{r}}\left(=\eta / \eta_{\mathrm{s}}\right)$ is the relative viscosity, $\eta$ and $\eta_{\mathrm{s}}$ are viscosities of the colloid and the solvent. The behavior of the OA colloid is very different from that of the other colloids with spherical particles, polystyrene beads (L1, L2), carbon black (CB), and rod-like titanate fibers ( $T F$, aspect ratio ca. 40 ${ }^{12)-15)}$. Systems which behave like the OA colloid have been never reported in the past. We consider that the OA molecule is spontaneously controlled (called auto-controlled) in order to suppress the increase in $\sigma_{\mathrm{y}}$ and viscosity with concentration.

This auto-controlled mechanism remains at other $\mathrm{pH}$ values. It is confirmed that the native $\mathrm{OA}$ aqueous colloids show marked yield stress and rigidity over a wide $\mathrm{pH}$ range of $3.2 \sim 7.1$. In Fig. 6 , values of $\sigma_{\mathrm{y}}$ are also plotted for the systems of $0.05 \%, \mathrm{pH}$ 4.46 and $9.1 \%, \mathrm{pH} 4.48$ (closed triangle). These $\mathrm{pH}$ values are near to the isoelectric point. The auto-controlled mechanism disappears with gelation due to heat denaturation as shown by half black circle in Fig. 6 (in the case of $70^{\circ} \mathrm{C}, 40$ min denaturation). At present, although there is no idea for the origin of the auto-controlled mechanism, we tried to apply the DLVO theory to obtain certain information.

\subsection{Application of DLVO theory}

According to the DLVO theory ${ }^{16)}$, the total potential energy of interaction $V_{t}$ between the colloidal particles is given by

$$
V_{\mathrm{t}}=V_{\mathrm{r}}+V_{\mathrm{a}}
$$

Here $V_{\mathrm{r}}$ and $V_{\mathrm{a}}$ are the potential energy due to the electrostatic repulsion and the van der Waals attraction, respectively. For spherical particles of radius $a$ with center-to-center separation distance $R$ and double layer poteintial $\psi_{\mathrm{d}}, V_{\mathrm{r}}$ is given by

$$
V_{\mathrm{r}}=\frac{4 \pi \varepsilon \varepsilon_{0} a^{2} \psi_{\mathrm{d}}^{2}}{R} \exp \{(-\kappa(R-2 a)\}
$$

where $\kappa$ is the Debye-Hückel parameter defined by

$$
\kappa=\left(\frac{2 \pi e^{2} L}{\varepsilon \varepsilon_{0} k T}\right)^{1 / 2}
$$

Here $L\left(=1 / 2 \sum n_{\mathrm{i}} Z_{\mathrm{i}}^{2}\right)$ is the ionic strength, $n_{\mathrm{i}}$ number of the $i$-th ion, $Z_{\mathrm{i}}$ the ionic valence, $\varepsilon_{0}$ the permittivity of free space, $\varepsilon$ the relative permittivity of the medium. The attraction term $V_{\mathrm{a}}$ is given by 


$$
V_{\mathrm{a}}=-\frac{A}{6}\left\{\frac{2}{s^{2}-4}+\frac{2}{s^{2}}+\ln \frac{s^{2}-4}{s^{2}}\right\}
$$

where $s=R / a$ and $A$ is the Hamaker constant, which is usually $10^{-21} \sim 10^{-18} \mathrm{~J}$. In the present case, the contribution of $V_{\mathrm{a}}$ is negligibly small. According to Buscall et al., shear modulus of the ordered structure is given by ${ }^{17)}$.

$$
\begin{aligned}
G_{\mathrm{th}}=4 \pi \alpha \varepsilon \varepsilon_{0} a^{2}{\psi_{\mathrm{d}}}^{2}\left(\frac{\kappa^{2} R^{2}+2 \kappa R+2}{R^{4}}\right) \\
\times \exp \{(-\kappa(R-2 a)\}
\end{aligned}
$$

Here $\alpha=0.833$ for fcc and 0.510 for bcc ordered arrangement.

The dashed line in Fig. 6, which is very different from the experimental result for the OA colloid (closed circle), is the concentration dependence of $G_{\mathrm{th}}$ calculated by Eq. (5) for $\kappa=3.12 \times 10^{-8} \mathrm{~m}^{-1}$ assuming the fcc arrangement. Here zeta-potential 21.6 $\mathrm{mV}$ at $\mathrm{pH} 7$ is used for $\psi_{\mathrm{d}}{ }^{8)}$. It is possible to make the calculated value coincide with the data by adjustment of $\kappa$ and the value of $\kappa$ is plotted against the OA concentration in Fig. 7 . The $k$ value is accurately proportional to $c^{1 / 2}$ over a wide concentration

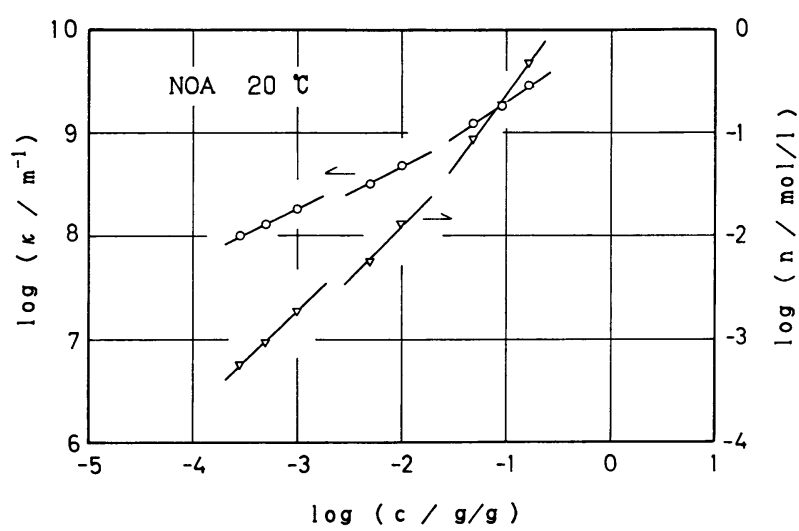

Fig. 7 The value of $\kappa$ and number of ions $n$, which is adjusted to make the thoeretical rigidity coincide with the experimental value, are plotted against the OA concentration range and the number of ions for monovalent ions is proportional to $c^{1}$. That is, if the OA colloid has some mechanism in which the concentration of the effective ions increases in proportion to the OA concentration, the curious behavior of the OA colloid in $\sigma_{\mathrm{y}}$ vs $\mathrm{c}$ shown in Fig. 6 can be explained in term of the electrostatic potential between the OA molecules. The OA content in egg-white is ca. $5.9 \%$. This value is approximately equal to $c^{*}$ in Fig. 6, i.e. the maximum concentration at which the OA colloid remains relative low rigidity and low viscosity and gelation due to heat denaturation does not occur. This suggests that the auto-controlled mechanism has some physiological or biological reasonableness in the natural state of the protein.

\section{References}

1) McReynolds, L., O'Malley, B.W., Nisbel, A.D., Fothergill, J.E., Givol, D., Fields, F., Robertson, M., Brownlee, G.G., Nature 273, 723 (1978).

2) Nisbet, A.D., Saundry, R.H., Moir, A.J., Fothergill, L.A., Fothergill, J.E., Fur J Biochem, 115, 335 (1981).

3) Tomimatsu, Y., Gaffield, W., Biopolym, 3, 509 (1965).

4) Gordon, J.A., J Biol Chem, 243, 4615 (1968).

5) Watanabe, K., New Food Ind, 24, 104 (1983).

6) Chen, Y.-H., Yang, J.T., Chau, K.H., Biochem, 13, 3350 (1974).

7) Matsumoto, T., Chiba, J., J Chem Soc Faraday Trans, 86, 2877 (1990).

8) Matsumoto, T., Chiba, J., Inoue, H., Coll Polym Sci, in press.

9) Koseki, T., Doi, E., Fukuda, T., Polym Prep Japan, 37, 1992 (1988).

10) Matsumoto, T., Segawa, Y., Warashina, Y., Onogi, S., Trans Soc Rheol, 17, 47 (1973).

11) Onogi, S., Matsumoto, T., Warashina, Y., Trans Soc Rheol, 17, 175 (1973).

12) Okubo, T., J Chem Soc Faraday Trans I, 85, 455 (1989).

13) Lindsay, H.H., Ghaikin, P.M., J Chem Phys, 76, 3774 (1982).

14) Mikami, Y., Onogi, S., J Soc Rheol Japan, 5, 110 (1977).

15) Onogi, S., Masuda, T., Matsumoto, T., Nippon Kagaku Zasshi, 88, 854 (1967).

16) Verwey, E.J.W., Overbeek, J.Th.G., “Theory of the Stability of Lyophobic Colloids", Elsevier Pub. London (1948).

17) Buscall, R., Goodwin, J.W., Hawkins, M.W., Ottewill, R.H., J Chem Soc Faraday Trans I, 78, 2889 (1982). 